\title{
The densest $k$-subgraph problem on clique graphs
}

\author{
M. Liazi ${ }^{1 \star}$, I. Milis $^{2}$, F. Pascual ${ }^{3}$ and V. Zissimopoulos ${ }^{1 \star \star}$ \\ 1 Department of Informatics and Telecommunications, \\ University of Athens, 15784 Athens, Greece \\ $\{$ mliazi,vassilis\}@di.uoa.gr \\ 2 Department of Informatics, \\ Athens University Economics and Business, \\ 10434 Athens, Greece \\ milis@aueb.gr \\ 3 IBISC, University of Évry, \\ 523, Place des Terrasses de l'agora, \\ 91000 Évry, France \\ fpascual@lami.univ-evry.fr
}

\begin{abstract}
The Densest $k$-Subgraph $(\mathrm{D} k \mathrm{~S})$ problem asks for a $k$-vertex subgraph of a given graph with the maximum number of edges. The problem is strongly NP-hard, as a generalization of the well known Clique problem and we also know that it does not admit a Polynomial Time Approximation Scheme (PTAS). In this paper we focus on special cases of the problem, with respect to the class of the input graph. Especially, towards the elucidation of the open questions concerning the complexity of the problem for interval graphs as well as its approximability for chordal graphs, we consider graphs having special clique graphs. We present a PTAS for stars of cliques and a dynamic programming algorithm for trees of cliques.
\end{abstract}

Key Words: Densest $k$-subgraph, Clique graph, Polynomial Time Approximation Scheme, Dynamic programming

\footnotetext{
* The project is co-financed within Op. Education by the ESF (European Social Fund) and National Resources.

** Partially supported by the Special Research Grants Account of the University of Athens under Grant 70/4/5821.
} 


\section{Introduction}

In the Densest $k$-subgraph $(\mathrm{D} k \mathrm{~S})$ problem we are given a graph $G=(V, E),|V|=n$, and an integer $k \leq n$, and we ask for a subgraph of $G$ induced by exactly $k$ of its vertices such that the number of edges of this subgraph is maximized. The problem is directly NP-hard as generalization of the well known Maximum Clique problem. In the weighted version of the $\mathrm{D} k \mathrm{~S}$ we also given non negative weights on the edges of $G$ and the goal is to find a $k$-vertex induced subgraph of maximum total edge weight.

During last years a large body of work $[2,3,5-7,10,14,19,20]$ has been concentrated on the design of approximation algorithms for both the $\mathrm{D} k \mathrm{~S}$ problem and its weighted version, based on a variety of techniques including greedy algorithms, LP relaxations and semidefinite programming. For a brief presentation of this body of work the reader is referred to the most recent of these articles [3]. However, the best known approximation ratio for the $\mathrm{D} k \mathrm{~S}$ problem, which performs well for all values of $k$, is $O\left(n^{\delta}\right)$, for some $\delta<\frac{1}{3}[5]$. On the other hand, it has been shown that the $\mathrm{D} k \mathrm{~S}$ problems does not admit a Polynomial Time Approximation Scheme (PTAS) [13]. However, there is not a negative result that achieving an approximation ratio of $O\left(n^{\epsilon}\right)$, for some $\epsilon>0$, is NP-hard. Concerning approximation algorithms for special cases of the problem it is known that the $\mathrm{D} k \mathrm{~S}$ problem admits a PTAS for graphs of minimum degree $\Omega(n)$ as well as for dense graphs (of $\Omega\left(n^{2}\right)$ edges) when $k$ is $\Omega(n)$ [1]. Moreover, algorithms achieving approximation factors of 4 [18] and 2 [11] have been proposed for the weighted $\mathrm{D} k \mathrm{~S}$ problem on complete graphs where the weights satisfy the triangle inequality.

The $\mathrm{D} k \mathrm{~S}$ problem is trivial on trees (any subtree of $k$ vertices contains exactly $k-1$ edges). It is also known that $\mathrm{D} k \mathrm{~S}$ is polynomial for graphs of maximal degree two [7] as well as for cographs, split graphs and k-trees [4]. On the other hand the DkS problem remains NP-hard for bipartite graphs [4], even of maximum degree three [7], as well as for comparability graphs, chordal graphs [4] and planar graphs [12]. The weighted version of 
the $\mathrm{D} k \mathrm{~S}$ problem is polynomial on trees either if we ask for a connected solution $[9,15,16]$ or not [17]. In fact, the result for the later case is implied by a result for the solution of the quadratic 0-1 knapsack problem on edge series-parallel graphs in [17].

An outstanding open question concerns the complexity of the $\mathrm{D} k \mathrm{~S}$ problem on interval graphs as well as its approximability for chordal graphs. Towards this direction we focus, in this paper, on chordal or interval graphs having special clique graphs. A clique of an undirected graph, $G=(V, E)$, is a subset of its vertices inducing a complete subgraph in $G$. The intersection graph of a family, $F$, of subsets of a set is defined as a graph, $\mathcal{G}$, whose vertices correspond to the subsets in $F$, and there is an edge between two vertices of $\mathcal{G}$ if the corresponding pair of subsets intersect. Given these definitions, the clique graph of a graph $G$ is defined as the intersection graph of the maximal cliques of $G$. It is well known that all maximal cliques, and hence the clique graph, of a chordal graph can be found in polynomial time [8]. It is, clearly, convenient to study the D $k \mathrm{~S}$ problem on the clique graph of a chordal graph $G$ instead on the $G$ itself.

In this paper we consider chordal or interval graphs having special clique graphs, in order to further identify the frontier between hard and polynomial solvable/approximable variants of the the $\mathrm{D} k \mathrm{~S}$ problem. In the next section we present a PTAS for graphs that having as clique graph a star (star of cliques) and in Section 3 we present an $O\left(n k^{m+1}\right)$ time dynamic programming algorithm for graphs having as clique graph a tree (tree of cliques) of maximum degree $m$. This algorithm gives an $O\left(n k^{3}\right)$ time algorithm for graphs having as clique graph a path (path of cliques). Note that, in general, stars of cliques as well as trees of cliques are neither graphs of minimum degree $\Omega(n)$ nor dense graphs (of $\Omega\left(n^{2}\right)$ edges) for which a PTAS is already known [1]. 


\section{The $\mathrm{D} k \mathrm{~S}$ problem on stars of cliques}

In this section we study graphs having as clique graph a star of cliques. Let $C_{0}, C_{1}, \ldots, C_{m-1}$ be the maximal cliques of such a star such that $C_{0}$ intersects with each other clique and no other intersection exists (by convention we denote by $C_{i}$ both the clique $C_{i}$ and the set of its vertices). Since such a star is the clique graph of a graph $G$, there is no edge of $G$ between vertices belonging to different cliques.

We shall call the $C_{0}$ central clique and all other cliques, $C_{i}, 1 \leq i \leq m-1$, exterior cliques. For each exterior clique $C_{i}$ we denote by $a_{i}$ the number of vertices in its intersection with $C_{0}$ i.e., $a_{i}=\left|C_{i} \cap C_{0}\right|$ and by $b_{i}$ the number of its vertices outside $C_{0}$ i.e., $b_{i}=\left|C_{i}\right|-a_{i}>$ 0 . By $C_{0}^{\prime}$ we denote the clique consisting of the vertices of $C_{0}$ not belonging to any other clique i.e., $C_{0}^{\prime}=C_{0} \backslash \bigcup_{i=1}^{m-1} C_{i}$.

By $S$ we denote a solution to the $\mathrm{D} k \mathrm{~S}$ problem i.e., a subset of $|S|=k$ vertices, and by $E(S)$ we denote the number of edges in the subgraph induced by $S$. By $S^{*}$ we denote an optimal solution to the $\mathrm{D} k \mathrm{~S}$ problem. By $n>k$ is denoted the total number of vertices in all cliques.

We say that a clique $C_{i}, 0 \leq i \leq m-1$, is completely in a solution $S$ if all its vertices are in $S$. On the other hand, we say that the cliques $C_{0}$ and $C_{0}^{\prime}$ are partially in a solution $S$ if a non-empty subset of their vertices, but not all, are in $S$. However, we say that an exterior clique $C_{i}, 1 \leq i \leq m-1$, is partially in $S$ if a non-empty subset of its $C_{i} \backslash C_{0}$ vertices, but not all, are in $S$. We distinguish the definition of the partial inclusion in a solution $S$ for an exterior clique $C_{i}$ because if only some of its $C_{i} \cap C_{0}$ vertices are in $S$, they can be considered as vertices of $C_{0}$. In general we say that a clique is participating in a solution $S$ if it is either completely or partially in $S$.

Concerning an optimal solution $S^{*}$ we observe that if an exterior clique $C_{i}$ is partially in $S^{*}$, then all its $\left|C_{i} \cap C_{0}\right|=a_{i}$ vertices are in $S^{*}$. Otherwise replacing a vertex $y \in$ $C_{i} \backslash C_{0}, y \in S^{*}$ by a vertex $x \in C_{i} \cap C_{0}, x \notin S^{*}$ yields a better solution, a contradiction. 
In the following we assume that:

(i) $k>\left|C_{i}\right|, i=0,1, \ldots, m-1$. Otherwise $S^{*}$ consists of any subset of $k$ vertices of some clique for which $\left|C_{i}\right| \geq k$.

(ii) $m>2$. For $m=1$ the point (i) holds. For $m=2$, if $k>\left|C_{0}\right| \geq\left|C_{1}\right|$, then $S^{*}$ consists of the vertices of $C_{0}$ plus any subset of $k-\left|C_{0}\right|$ vertices of $C_{1} \backslash C_{0}$.

Using these definitions and assumptions we give in the next propositions some structural properties of an optimal solution $S^{*}$.

Proposition 1. At most one of the cliques $C_{0}^{\prime}, C_{1}, \ldots, C_{m-1}$ is partially in an optimal solution.

proof: We prove first that at most one of the exterior cliques is partially in $S^{*}$. Suppose that two exterior cliques $C_{i}, C_{j}, 1 \leq i \neq j \leq m-1$ are partially in $S^{*}$ and assume w.l.o.g. that $\left|S^{*} \cap C_{i}\right| \geq\left|S^{*} \cap C_{j}\right|$.

Let $x \notin S^{*}$ be a vertex in $C_{i} \backslash C_{0}$ and $y \in S^{*}$ be a vertex in $C_{j} \backslash C_{0}$. Then consider the solution $S$ in which we replace $y$ by $x$. Then, $E(S)=E\left(S^{*}\right)-\left(\left|S^{*} \cap C_{j}\right|-1\right)+\left|S^{*} \cap C_{i}\right| \geq$ $E\left(S^{*}\right)+1$, a contradiction to the optimality of $S^{*}$.

To complete the proof it suffices to prove that is not possible both clique $C_{0}^{\prime}$ and an exterior clique $C_{j}$ to be partially in $S^{*}$. This fact follows by using the same arguments as before, but now we consider $C_{0}$ instead of $C_{i}$ and $x \notin S^{*}$ to be a vertex in $C_{0}^{\prime}$.

\section{Proposition 2.}

(i) If $C_{0}$ is the largest clique i.e., $\left|C_{0}\right|>\left|C_{i}\right|, 1 \leq i \leq m-1$, then $C_{0}$ belongs completely to every optimal solution.

(ii) If $C_{0}$ is partially in an optimal solution $S^{*}$, then $\left|C_{0}\right| \leq\left|C_{i}\right|$ for every clique $C_{i}$ participating in $S^{*}$.

proof: 
(i) Suppose that $S^{*}$ does not contain some $q>0$ vertices of $C_{0}$ and consider a solution $S$ obtained from $S^{*}$ by replacing $q$ vertices of exterior cliques not in $C_{0}$ by the $q$ vertices of $C_{0}$ not in $S^{*}$. Let us denote by $E^{-}$and $E^{+}$the number of edges which are removed and inserted, respectively, to $E\left(S^{*}\right)$ by this replacement. Then, $E(S)=E\left(S^{*}\right)-E^{-}+E^{+}$. $E^{-}$equals to the number of edges that $q$ vertices of exterior cliques contribute to $E\left(S^{*}\right)$. This number, even if all the $q$ vertices belong to the same exterior clique, is strictly less than $\left(\begin{array}{l}q \\ 2\end{array}\right)+\left(\left|C_{0}\right|-q\right) q$. On the other hand, $E^{+}$equals to the number of edges that the $q$ vertices of $C_{0}$ not in $S^{*}$ will contribute to $E(S)$. This number is equal to $\left(\begin{array}{l}q \\ 2\end{array}\right)+\left(\left|C_{0}\right|-q\right) q$. Therefore, $E(S)>E\left(S^{*}\right)$, a contradiction to the optimality of $S^{*}$.

(ii) Suppose that there is an exterior clique $C_{i}$ in $S^{*}$ such that $\left|C_{0}\right|>\left|C_{i}\right|$ and that $S^{*}$ does not contain some $q>0$ vertices of $C_{0}$. Notice that $\left|C_{0}\right|-q>a_{i}$, since if $\left|C_{0}\right|-q \leq a_{i}$, then no other exterior clique participates in $S^{*}$, that is $S^{*}$ is part of a single clique (either $C_{i}$ or $\left.C_{0}\right)$.

Consider a solution $S$ obtained from $S^{*}$ by replacing vertices of $S^{*} \cap C_{i}$ not in $C_{0}$ by vertices of $C_{0}$ not in $S^{*}$. Let $b_{i}^{\prime}=\left|S^{*} \cap\left(C_{i} \backslash C_{0}\right)\right|, 0<b_{i}^{\prime} \leq b_{i}$. Using again $E^{-}$and $E^{+}$ as in part (i) we have $E(S)=E\left(S^{*}\right)-E^{-}+E^{+}$. Now we distinguish between two cases w.r.t. the values of $q$ and $b_{i}^{\prime}$.

If $q \geq b_{i}^{\prime}$ then $E^{-}$equals to the number of edges that $b_{i}^{\prime}$ vertices of the exterior clique $C_{i}$ contributes to $E\left(S^{*}\right)$ while $E^{+}$equals to the number of edges that the $b_{i}^{\prime}$ vertices of $C_{0}$ not in $S^{*}$ will contribute to $E(S)$. Then $E(S)=E\left(S^{*}\right)-E^{-}+E^{+}=E\left(S^{*}\right)-\left(\left(\begin{array}{c}b_{i}^{\prime} \\ 2\end{array}\right)+\right.$ $\left.b_{i}^{\prime} a_{i}\right)+\left(\left(\begin{array}{c}b_{i}^{\prime} \\ 2\end{array}\right)+b_{i}^{\prime}\left(\left|C_{0}\right|-q\right)\right)=E\left(S^{*}\right)+b_{i}^{\prime}\left(\left(\left|C_{0}\right|-q\right)-a_{i}\right)>E\left(S^{*}\right)$, a contradiction to the optimality of $S^{*}$.

If $q<b_{i}^{\prime}$ then $E^{-}$equals to the number of edges that $q$ vertices of the exterior clique $C_{i}$ contributes to $E\left(S^{*}\right)$ while $E^{+}$equals to the number of edges that the $q$ vertices of $C_{0}$ not in $S^{*}$ will contribute to $E(S)$. Then $E(S)=E\left(S^{*}\right)-E^{-}+E^{+}=E\left(S^{*}\right)-\left(\left(\begin{array}{c}q \\ 2\end{array}\right)+q\left(a_{i}+b_{i}^{\prime}-\right.\right.$ 
$q))+\left(\left(\begin{array}{l}q \\ 2\end{array}\right)+q\left(\left|C_{0}\right|-q\right)\right)=E\left(S^{*}\right)+q\left(\left|C_{0}\right|-\left(a_{i}+b_{i}^{\prime}\right)\right) \geq E\left(S^{*}\right)+q\left(\left|C_{0}\right|-\left|C_{i}\right|\right)>E\left(S^{*}\right), \mathrm{a}$ contradiction to the optimality of $S^{*}$.

Despite the nice structural properties of an optimal solution in Propositions 1 and 2, many natural greedy criteria based on the sizes of the cliques or/and the sizes of intersections fail to give such an optimal solution. In the following we are able to give a polynomial time dynamic programming algorithm for the case where the central clique is completely in the optimal solution and a PTAS for the general case.

\subsection{Clique $C_{0}$ is completely in the optimal solution.}

Lemma 1. If clique $C_{0}$ is completely in the optimal solution, then there is an $O\left(n k^{2}\right)$ dynamic programming algorithm for the DkS problem on a star of cliques.

proof: Since clique $C_{0}$ is completely in the optimal solution we have to choose $k^{\prime}=k-\left|C_{0}\right|$ vertices from exterior cliques. If we choose $q$ vertices from an exterior clique $C_{j}$, then they contribute $q \cdot a_{j}+\left(\begin{array}{c}q \\ 2\end{array}\right)$ edges to the solution.

Let $f(i, j)$ be the maximum number of edges in a solution choosing $i$ vertices from the first $j$ exterior cliques (recall that there are $m-1$ exterior cliques). Thus for $i=0,1,2, \ldots, k^{\prime}$ and $j=2,3, \ldots, m-1$

$$
f(i, j)=\max _{0 \leq q \leq \min \left\{i, b_{j}\right\}}\left\{f(i-q, j-1)+q \cdot a_{j}+\left(\begin{array}{l}
q \\
2
\end{array}\right)\right\}
$$

For $j=1$ the following boundary conditions hold for $0 \leq i \leq \min \left\{k^{\prime}, b_{1}\right\}$

$$
f(i, 1)= \begin{cases}\left(\begin{array}{l}
i \\
2
\end{array}\right)+i \cdot a_{1}, & \text { if } i \leq \min \left\{k^{\prime}, b_{1}\right\} \\
-\infty, & \text { otherwise }\end{cases}
$$


The complexity of the dynamic programming algorithm is $O\left(n k^{2}\right)$. The computation of a single $f(i, j)$ value takes $O(k)$ time due to the possible values of $q\left(0 \leq q \leq \min \left\{i, b_{j}\right\} \leq\right.$ $\left.k^{\prime}<k\right)$ and $f(i, j)$ values are computed for every $i \leq k^{\prime}<k$ and $j \leq m-1<n$. The optimal solution, for the $\mathrm{D} k \mathrm{~S}$ problem is $f\left(k^{\prime}, m-1\right)+\left(\begin{array}{c}\left|C_{0}\right| \\ 2\end{array}\right)$.

Notice that if $C_{0}$ is the largest clique, then, by Proposition 2(i), $C_{0}$ belongs completely to every optimal solution and the above dynamic programming algorithm applies.

\subsection{A PTAS for the general case.}

In the general case, $C_{0}$ is partially in the optimal solution and, by Proposition 2(i), there are exterior cliques larger than $C_{0}$. Let $c$ be the number of those cliques of size at least $\left|C_{0}\right|$. Moreover, by Proposition 2(ii), the cliques participating in the optimal solution are some of these $c$ cliques. Next proposition gives a weak upper bound for the number $c$.

Proposition 3. If $C_{0}$ is partially in an optimal solution, then the number of exterior cliques of size at least $\left|C_{0}\right|$ is at most $\sqrt{n}$.

proof: The number, $c$, of exterior cliques is smaller than or equal to $\left|C_{0}\right|$, since $C_{i} \cap C_{0} \neq \emptyset$ and $C_{j} \cap C_{i}=\emptyset, 1 \leq i \neq j \leq m-1$. Thus, if $\left|C_{0}\right| \leq \sqrt{n}$, then $c \leq \sqrt{n}$. Otherwise $\left|C_{0}\right|>\sqrt{n}$. Then, the total number of vertices in these $c$ cliques is at least $c \times \sqrt{n}$ and at most $n$. Hence, $c \leq \sqrt{n}$.

To proceed towards a PTAS we argue further on the number of the exterior cliques of size at least $\left|C_{0}\right|$. We define $r=\left\lfloor\frac{k}{\left|C_{0}\right|}\right\rfloor$. Then the number of exterior cliques of size at least $\left|C_{0}\right|$ that can be involved in an optimal solution is at most $r$. Let also $\delta$ be a fixed number which will be defined later. Comparing $r$ with $\delta$ we distinguish between two cases. 
Case 1: $r<\delta$

If $r$ is "small", then we proceed in an exhaustive manner. We examine all the possible sets of $r$ cliques out of $c$ cliques of size at least $\left|C_{0}\right|$ i.e., $\left(\begin{array}{l}c \\ r\end{array}\right)$ sets of cliques. A technical detail here is that clique $C_{0}^{\prime}$ should be also considered as one of the $c$ cliques. It can be easily done by considering clique $C_{0}^{\prime}$ as an external clique with zero vertices outside clique $C_{0}$.

By Proposition 3 it follows that the number of all the $\left(\begin{array}{l}c \\ r\end{array}\right)$ sets of cliques is $O\left(n^{\frac{r}{2}}\right)$. For each one of these sets of $r$ cliques we compute the $k$ vertices that maximize the number of edges as follows:

Let $R$ be a set of $r$ cliques. By Proposition 1, at most one of the cliques in $R$ is partially in $S^{*}$. We consider all the $2^{r}-1$ subsets of $R$. Let $R_{i}$ be one of these subsets and let $C_{i}^{j}$ be the $j^{\text {th }}, 1 \leq j \leq\left|R_{i}\right|$, clique of the set $R_{i}$. Clearly if $\sum_{j=1}^{\left|R_{i}\right|}\left|C_{i}^{j}\right|<k$, we discard the set $R_{i}$. Otherwise, let $k(j)=\sum_{t=1, t \neq j}^{\left|R_{i}\right|}\left|C_{i}^{t}\right|$, for each $j=1,2, \ldots,\left|R_{i}\right|$. If $k(j)>k$ then we discard this $j$. Otherwise (if $k(j) \leq k$ ) we obtain a $k$-vertex solution by taking $k-k(j)$ vertices from clique $C_{i}^{j}$, starting from vertices which belong to its intersection with $C_{0}$.

Consider now all the solutions obtained for each $j=1,2, \ldots,\left|R_{i}\right|$, and for each $R_{i} \subseteq R$. By their construction, these solutions are all the possible $k$-vertex solutions for the set $R$ of cliques, under the restriction that at most one of them is partially taken. Therefore, to find the optimal solution we simply have to choose the one with the maximum number of edges.

For a set $R$ of $r$ cliques, there are $2^{r}-1$ subsets $R_{i}$, and for each subset there are at most $r$ possible solutions. Therefore, the number of solutions to compare is $O\left(r 2^{r}\right)$.

Recalling that we have to examine $O\left(n^{\frac{r}{2}}\right)$ sets of $r$ cliques, the next lemma follows.

Lemma 2. For the case $r<\delta, \delta$ be a fixed number, an optimal solution for the DkS problem in a star of cliques can be found in $O\left(r 2^{r} n^{\frac{r}{2}}\right)$ time. 
Case 2: $r \geq \delta$

If $r$ is "large", then we proceed in a greedy manner. We consider the solution, $S$, obtained by the following simple algorithm:

Let $C_{1} \geq C_{2} \geq \ldots \geq C_{m-1}$ and $t$ be the largest integer number such that $k \geq \sum_{i=1}^{t}\left|C_{i}\right|=$ $k^{\prime}$.

Return all the vertices of the cliques $C_{1} \geq C_{2} \geq \ldots \geq C_{t}$ and $k-k^{\prime}$ vertices of clique $C_{t+1}$.

Next proposition for the case of independent cliques will be useful for bounding the deviation of our solution from the optimal one.

Proposition 4. Let $R_{1}$ and $R_{2}$ be two sets of independent cliques with all cliques in $R_{1}$ of size at least $L$ and all cliques in $R_{2}$ of size exactly $L$. For any pair of sets of $k$ vertices $S_{1}$ and $S_{2}$ in $R_{1}$ and $R_{2}$, respectively, such that in both sets at most one clique is taken partially, it holds that $E\left(S_{1}\right) \geq E\left(S_{2}\right)$.

proof: Transform $S_{1}$ to an equivalent to $S_{2}$ set as following. First, remove from each clique in $S_{1}$ some vertices such that each clique in $S_{1}$ has now size exactly $L$. Let $k^{\prime}$ the number of the removed vertices. Then, replace the $k^{\prime}$ vertices with $\left\lceil\frac{k^{\prime}}{L}\right\rceil$ cliques, all, but one, of size exactly $L$. All the removed vertices now have degree at most $L-1$ while in $S_{1}$ they had degree at least $L-1$. Thus, $E\left(S_{1}\right) \geq E\left(S_{2}\right)$.

Let us now consider the solution $S$ obtained by our algorithm. By Proposition 2(ii), the optimal solution $S^{*}$ involves exterior cliques of size at least $\left|C_{0}\right|$. Since our algorithm finds a solution $S$ by choosing $k$ vertices from the larger exterior cliques, it follows that all cliques in $S$ are of size at least $\left|C_{0}\right|$. 
Moreover, since $r=\left\lfloor\frac{k}{\left|C_{0}\right|}\right\rfloor$, we need at least $r$ cliques of size $\left|C_{0}\right|$ in order to fill $k$. Hence, choosing $k$ vertices from a set of independent cliques of size $\left|C_{0}\right|$, yields at least $r E\left(C_{0}\right)$ edges. Therefore, by Proposition 4, it follows that $E(S) \geq r E\left(C_{0}\right)$.

Clearly, an optimal solution, $S^{*}$, could contain cliques of smaller size than those chosen by our algorithm. These small cliques are selected by $S^{*}$ due to the edges between their overlaps with $C_{0}$. Since these edges belong to $C_{0}$, the optimal solution cannot be greater than $E(S)$ plus the edges of $C_{0}$ i.e.,

$$
E\left(S^{*}\right) \leq E(S)+E\left(C_{0}\right) \leq E(S)+\frac{E(S)}{r} \leq E(S)+\frac{E(S)}{\delta}=E(S) \frac{\delta+1}{\delta} .
$$

Thus, next lemma follows.

Lemma 3. For the case $r \geq \delta$, where $\delta=\frac{1-\epsilon}{\epsilon}, 0<\epsilon<1$, there is an $(1-\epsilon)$-approximation algorithm for the DkS problem in a star of cliques.

The complexity of the greedy approximation algorithm of Lemma 3 is $O(n \log n)$. The complexity of the exhaustive optimal algorithm of Lemma 2 is exponential in $r \leq \delta=\frac{1-\epsilon}{\epsilon}$, that is exponential in $\frac{1}{\epsilon}$. Hence, we obtain

Theorem 1. There is a polynomial time approximation scheme for the DkS problem in stars of cliques.

\section{The DkS problem on trees of cliques.}

In this section we present a dynamic programming algorithm which yields an optimal solution for the $\mathrm{D} k \mathrm{~S}$ problem for graphs having as clique graph a tree. Let $C_{1}, C_{2}, \ldots, C_{t}$ be the cliques of such a tree and $m$ its maximum degree. We consider $\left|C_{i}\right|<k, i=1, \ldots, t$, otherwise the problem is trivial. 
We consider the tree rooted at a leaf clique, say clique $C_{t}$. This way the root clique $C_{t}$ has at least one vertex outside its intersections with its children cliques. Let $C_{i}$ be a non-leaf clique with $m_{i} \geq 1$ children, $C_{i_{1}}, \ldots, C_{i_{m_{i}}}$. We denote by $Q_{h}$ the intersection of $C_{i}$ with its $h^{\text {th }}$ child clique, $C_{h}$, for $h=i_{1}, \ldots, i_{m_{i}}$ i.e., $Q_{h}=C_{i} \cap C_{h}$. We denote by $F_{i}$ the intersection of the clique $C_{i}$ with its father clique, $C_{f}$, in the tree i.e., $F_{i}=C_{i} \cap C_{f}$ and by $D_{i}$ the vertices of a clique $C_{i}$ not belonging to any intersection i.e., $D_{i}=C_{i}-F_{i}-\bigcup_{h=i_{1}}^{i_{m_{i}}} Q_{h}$. By convention we consider $F_{t}$, for the root clique $C_{t}$, to be consisted of a single vertex i.e., $\left|F_{t}\right|=1$.

The algorithm traverses the tree of cliques starting from the leaves cliques. In each step it computes an optimal solution for all the $j$-vertex densest subgraph $(\mathrm{D} j \mathrm{~S})$ problems, for $j=1, \ldots, k$, on the subtree rooted at clique $C_{i}$.

We denote by $f_{i}(j)$ the value of the optimal solution of the $\mathrm{D} j \mathrm{~S}$ problem on the subtree rooted at clique $C_{i}$. By $f_{i}(j, a)$ we denote the value of an optimal solution to the $\mathrm{D} j \mathrm{~S}$ problem on the subtree rooted at clique $C_{i}$ including exactly a vertices from the clique $F_{i}$. It is clear that

$$
f_{i}(j)=\max _{0 \leq a \leq\left|F_{i}\right|}\left\{f_{i}(j, a)\right\}
$$

To compute an $f_{i}(j, a)$ value for a non leave clique $C_{i}$ we consider its children cliques $C_{i_{1}}, \ldots, C_{i_{m_{i}}}, m_{i} \geq 1$. Let $f_{h}\left(j_{h}, a_{h}\right)$ be the value of an optimal solution of the $j_{h}$-vertex densest subgraph, for $j_{h}=1,2, \ldots, k$, on the subtree rooted at clique $C_{h}, h=i_{1}, \ldots, i_{m_{i}}$, using $a_{h}$ vertices of $F_{h}$. Note that for the intersection of the clique $C_{i}$ with its child $C_{h}$ it holds that $Q_{h}=C_{i} \cap C_{h}=F_{h}$. We compute the value of $f_{i}(j, a)$, as follows:

If $a=0$, then no vertex of $F_{i} \cup D_{i}$ belongs to the optimal solution of the corresponding $\mathrm{D} j \mathrm{~S}$ problem on the subtree rooted at $C_{i}$. Therefore, the value of this solution is the same as the value of the optimal solution to the $\mathrm{D} j \mathrm{~S}$ problem on the subgraph which is the union of the subtrees rooted at the children of $C_{i}$ plus the edges between the vertices in their 
$Q_{h}$ 's, that is

$$
f_{i}(j, a)=\max _{\sum_{h=i_{1}}^{i_{m_{i}}} j_{h}=j}\left\{\sum_{h=i_{1}}^{i_{m_{i}}} f_{h}\left(j_{h}, a_{h}\right)+\sum_{\substack{i, j=i_{1} \\ i \neq j}}^{i_{m_{i}}} \frac{a_{i} \cdot a_{j}}{2}\right\}
$$

If $a>0$, then an optimal solution to the $\mathrm{D} j \mathrm{~S}$ problem including $a$ vertices of $F_{i}$ can also include $b \geq 0$ vertices of $D_{i}$, and $a_{h} \geq 1$ vertices ${ }^{4}$ of each $Q_{h}, h=i_{1}, \ldots, i_{m_{i}}$. Then,

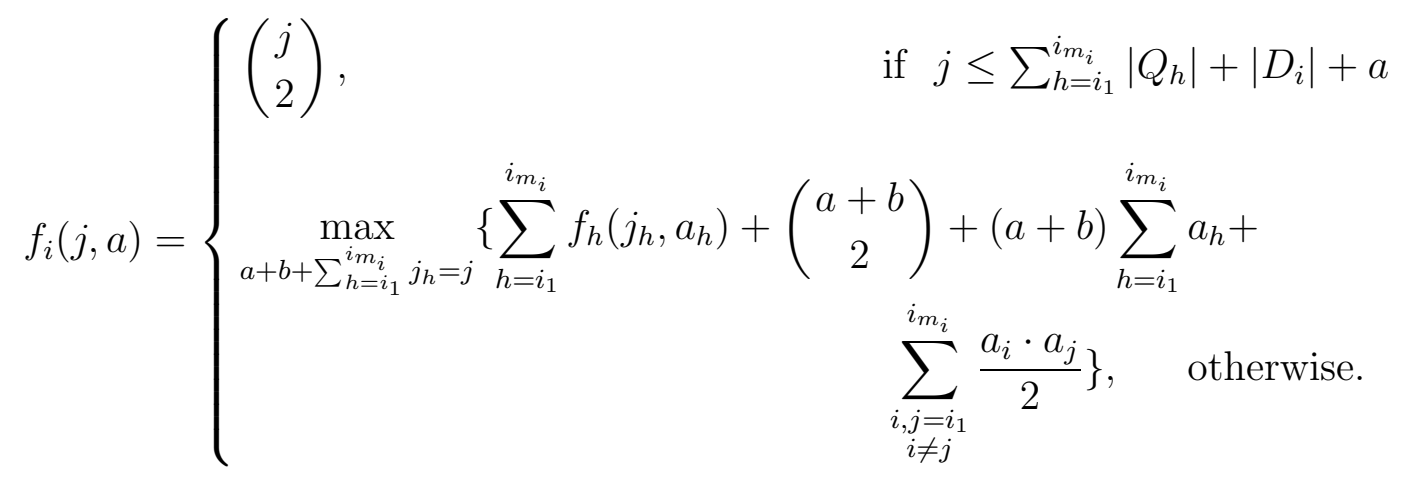

For all cliques $C_{i}$ that are leaves in the tree the following boundary conditions hold for $1 \leq j \leq k$ :

If $a=0$, then $f_{i}(j, a)=0$.

If $1 \leq a \leq\left|F_{i}\right|$, then

$$
f_{i}(j, a)= \begin{cases}\left(\begin{array}{c}
j \\
2
\end{array}\right), & \text { if } j \leq\left|D_{i}\right|+a \\
-\infty, & \text { otherwise. }\end{cases}
$$

The algorithm terminates by computing the value $f_{t}(k)$ for the root clique $C_{t}$. Recall that we consider $\left|F_{t}\right|=1$ and thus the optimal solution for the $k$-vertex densest subgraph problem is $f_{t}(k)=\max _{a=0,1}\left\{f_{t}(k, a)\right\}$.

The computation of a single $f_{i}(j)$ value for a clique $C_{i}$ with $m_{i}$ children takes $O\left(k^{m_{i}+1}\right)$ time due to the combinations of $a, b$ and $\sum_{h=i_{1}}^{i_{m_{i}}} j_{h}$, such that $a+b+\sum_{h=i_{1}}^{i_{m_{i}}} j_{h}=j$. The 
algorithm computes $f_{i}(j)$, for every $j=1,2, \ldots, k$ and for every $i=1,2, \ldots, t$. Since in the worst case $t$ is $O(n)$ and $\max _{i}\left\{m_{i}\right\}=m-1$ the next theorem follows:

Theorem 2. There is an $O\left(n k^{m+1}\right)$ algorithm for the DkS problem on a tree of cliques of maximum degree $m$.

Next corollary follows directly from Theorem 2 .

Corollary 1. There is an $O\left(n k^{3}\right)$ optimal algorithm for the DkS problem on a path of cliques.

\section{Conclusions}

We have presented a PTAS for the densest $k$-subgraph problem on a star of cliques and an $O\left(n k^{m+1}\right)$ time optimal algorithm for the same problem on trees of cliques, where $n$ is the total number of vertices in all the cliques and $m$ the maximum degree of the tree. This last algorithm gives an $O\left(n k^{3}\right)$ optimal algorithm for paths of cliques. Since interval and chordal graphs can be seen as clique graphs our result could be exploited in the direction of exploring the complexity and the approximability of the $\mathrm{D} k \mathrm{~S}$ problem in these classes of graphs.

Acknowledgment: The authors would like to thank the two anonymous referees for their valuable comments that significantly improved the presentation.

\section{References}

1. S. Arora, D. Karger, and M. Karpinski. Polynomial time approximation schemes for dense instances of NP-hard problems. In Proceedings of the 27th annual ACM symposium on Theory of Computing, pages 284-293, 1995.

2. Y. Asahiro, K. Iwama, H. Tamaki, and T. Tokuyama. Greedily finding a dense subgraph. Journal of Algorithms, $34(2): 203-221,2000$. 
3. A. Billionnet and F. Roupin. A deterministic algorithm for the densest k-subgraph problem using linear programming. Technical Report No486, CEDRIC, CNAM-IIE, Paris, 2004.

4. D. G. Corneil and Y. Perl. Clustering and domination in perfect graphs. Discrete Applied Mathematics, 9:27-39, 1984 .

5. U. Feige, G. Kortsarz, and D. Peleg. The dense k-subgraph problem. Algorithmica, 29(3):410-421, 2001.

6. U. Feige and M. Langberg. Approximation algorithms for maximization problems arising in graph partitioning. Journal of Algorithms, 41(2):174-211, 2001.

7. U. Feige and M. Seltser. On the densest k-subgraph problem. Technical Report CS97-16, Weizmann Institute, 1997.

8. F. Gavril. Algorithms for minimum coloring, maximum clique, minimum covering by cliques and maximum independent set of chordal graph. SIAM Journal on Computing, 1(2):180-187, 1972.

9. O. Goldschmidt and D. Hochbaum. k-edge subgraph problems. Discrete Applied Mathematics and Combinatorial Operations Research and Computer Science, 74(2):159-169, 1997.

10. Q. Han, Y. Ye, and J. Zhang. An improved rounding method and semidefinite programming relaxation for graph partition. Mathematical Programming, 92(3):509-535, 2002.

11. R. Hassin, S. Rubinstein, and A. Tamir. Approximation algorithms for maximum dispersion. Operations Research Letters, 21(3):133-137, 1997.

12. J. M. Keil and T. B. Brecht. The complexity of clustering in planar graphs. Journal of Combinatorial Mathematics and Combinatorial Computing, 9:155-159, 1991.

13. S. Khot. Ruling out PTAS for graph min-bisection, densest subgraph and bipartite clique. In Proceedings of the 45th Annual IEEE Symposium on Foundations of Computer Science, pages 136-145, 2004.

14. G. Kortsarz and D. Peleg. On choosing a dense subgraph. In Proceedings of the 34th Annual IEEE Symposium on Foundations of Computer Science, pages 692-701, 1993.

15. F. Maffioli. Finding a best subtree of a tree. Technical Report 91.041, Politechnico di Milano, Dipartimento di Elektronica, 1991.

16. Y. Perl and Y. Shiloach. Efficient optimization of monotonic functions on trees. SIAM Journal of Algebric and Discrete Methods, 4(4):512-516, 1983.

17. D. J. Jr. Rader and G. J. Woeginger. The quadratic 0-1 knapsack problem with series-parallel support. Operation Research Letters, 30(3):159-166, 2002.

18. S. S. Ravi, D. J. Rosenkrantz, and G. K. Tayi. Heuristic and special case algorithms for dispersion problems. Operations Research, 42(2):299-310, 1994.

19. A. Srivastav and K. Wolf. Finding dense subgraphs with semidefinite programming. In Proceedings of the International Workshop on Approximation Algorithms for Combinatorial Optimization, pages 181-191, 1998.

20. Y. Ye and J. Zhang. .519 approximation of dense-n/2-subgraph. Working Paper, Department of Management Sciences, Henry B. Tippie College of Business, The University of Iowa, 1999. 